\title{
Diffused Quantum Well Solar Cells
}

\author{
Y. Cheng, Alex S.W. Lee and E. Herbert. Li \\ Department of Electrical and Electronic Engineering \\ The University of Hong Kong, Pokfulam Road, Hong Kong
}

\begin{abstract}
An alternative multi-bandgap solar cell made of diffused quantum well (DFQW) as the absorber is proposed here. The modeling of the spectral response and energy conversion efficiency of the solar cell will be shown. Significant enhancement in energy conversion efficiency is demonstrated when compared to that of the single bandgap cells.
\end{abstract}

\section{INTRODUCTION}

Incorporating quantum wells into the absorption layer of the solar cell as an alternative to conventional multi-bandgap approach has been proposed'. Significant enhancements in short circuit current and energy conversion efficiency have already been demonstrated by using variable well width AlGaAs/GaAs p-i-n multiquantum well (MQW) photodiodes ${ }^{2}$. The results show prospect for using QW as the active absorber in the solar cell. We propose here an alternative way of improving the efficiency by using diffused quantum well (DFQW) system with equal well width, which is presumably easier to fabricate than the QW structure with variable well width. The different bandgaps of the $\mathrm{QW}$ system can be selectively modified by employing suitable masks to control the extent of ion implanted into the required region. The efficiency of the DFQW cell compared to that of the single-band-gap is shown to be increased. The calculations on spectral response and energy conversion efficiency are based on ideal solar cell models.

\section{MODEL}

The proposed structure, depicted in Fig.1, consists of $\mathrm{Al}_{\mathrm{x}} \mathrm{Ga}_{1-\mathrm{x}} \mathrm{As} / \mathrm{GaAs}$ and $\mathrm{GaAs} / \mathrm{In}_{\mathrm{y}} \mathrm{Ga}_{1-\mathrm{y}} \mathrm{As} \mathrm{DFQW}$ system in the depletion region of a heterostructure solar cell. We choose $\mathrm{Al}_{\mathrm{x}} \mathrm{Ga}_{1-\mathrm{x}} \mathrm{As} / \mathrm{GaAs} \mathrm{QW}$ system because of its best understood well properties among the III-V materials. The choice of $\mathrm{GaAs} / \mathrm{In}_{\mathrm{y}} \mathrm{Ga}_{1-\mathrm{y}} \mathrm{As}$ is for a lower absorption bandgap range which results in an increase in the overall absorption wavelength and an extended spectral response.

In order to have a larger area of photon collection, the front contact of the solar cell is grooveetched. The DFQW system consists of 10 periods of barrier/well with the well width $=100 \AA$ and barrier width $=200 \AA$. A possible way to fabricate the proposed MQW system with different bandgaps is to use a mask during ion implantation as shown in Fig.2. After implantation, the mask shown in the figure will be removed.

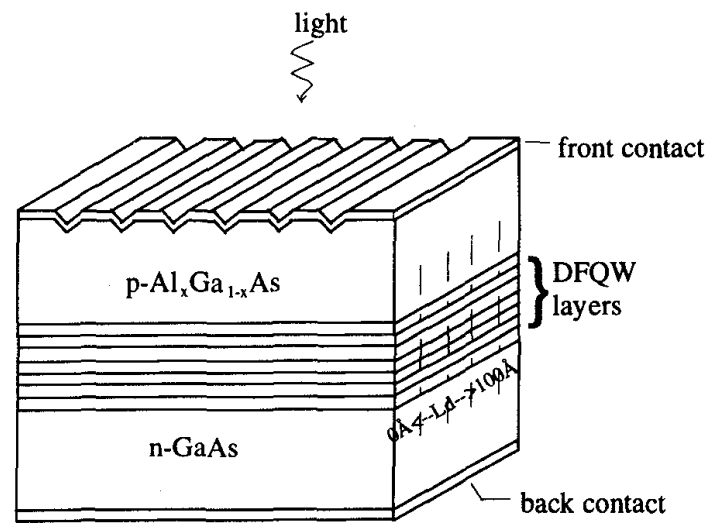

Fig.1. Schematic representation of a diffused quantum well solar cell. Bandgap energy of the DFQW layers varies as the diffusion length of the implanted ions.

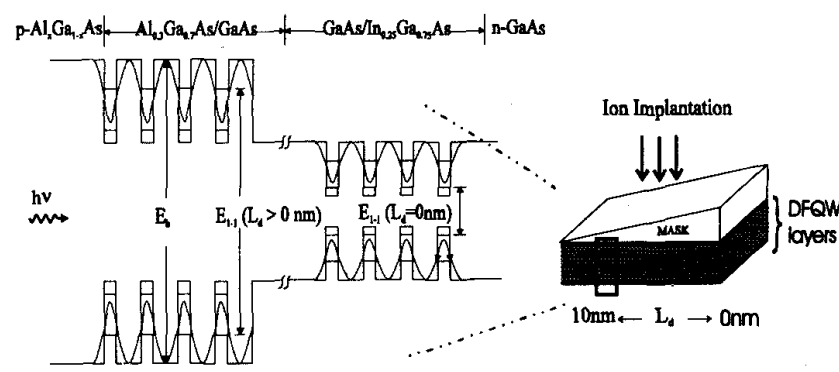

(a)

(b)

Fig.2. Schematic representation of the DFQW layers with well width $=10 \mathrm{~nm}$ and barrier $=20 \mathrm{~nm}$. (a) Bandgap structure is shown in 90 degrees rotation. Curve lines represent the bandgaps after diffusion. (b) The bandgaps are modified by using ion implantation process. 
The choice of aluminum mode fraction around 0.3 is from a common practice. The indium mode fraction $=0.25$ is to avoid reaching the critical layer thickness ${ }^{3}$. We let the barrier width doubles that of the well is to minimize the interference from adjacent wells so that we can use the single $\mathrm{QW}$ model to calculate the absorption coefficient.

In our system, the well width of $100 \AA$ is based on the maximum diffusion length of the implanted ions, $L_{d}$ ion $=100 \AA$ and is determined from the following MQW diffusion model $^{4}$ for the composition profile:

$$
\begin{aligned}
w(z)= & \frac{w_{o}}{2}\left\{\sum_{i=1}^{n}\left[\operatorname{erf}\left(\frac{z-a_{i}}{2 L_{d}}\right)-\operatorname{erf}\left(\frac{z-b_{i}}{2 L_{d}}\right)\right]\right. \\
& \left.+\left[2-e r f\left(\frac{z-c_{1}}{2 L_{d}}\right)-\operatorname{erf}\left(\frac{z-c_{2}}{2 L_{d}}\right)\right]\right\},
\end{aligned}
$$

where $\mathrm{w}_{0}$ is the as-grown $\mathrm{Al}$ (or In for InGaAs) concentration in both the barrier and the cladding layer; $\mathbf{n}$ is the number of as-grown barriers within the MQW core; $\operatorname{erf}(z)$ represents the error function; $a_{i}$ and $b_{i}$ represent the $\mathrm{i}^{\text {th }}$ as-grown barrier within the MQW core and are the left and right interface positions respectively; $c_{1}$ and $c_{2}$ are the position of the interfaces between the cladder and the core. In Fig. 3, we can see how the bandgaps vary with different diffusion length.

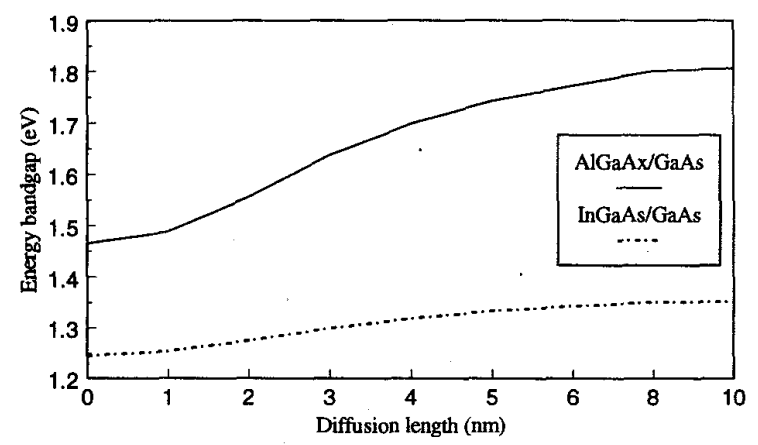

Fig. 3. Energy bandgap varies as a function of diffusion length.

The spectral response was first calculated by solving the minority carriers continuity equations in a fieldness model ${ }^{s}$ at room temperature to obtain the photocurrent $J_{n}$ and $J_{p}$ from the $p$ and $n$ region.

$$
\begin{aligned}
& D_{p} \frac{d^{2} \Delta n_{p}}{d x^{2}}+\alpha F \exp (-\alpha x)-\frac{\Delta n_{p}}{\tau_{n}}=0, \\
& D_{n} \frac{d^{2} \Delta p_{n}}{d x^{2}}+\alpha F \exp (-\alpha x)-\frac{\Delta p_{n}}{\tau_{p}}=0,
\end{aligned}
$$

The boundary conditions for p-type material, $\mathrm{Al}_{\mathrm{x}} \mathrm{Ga}_{1-x} \mathrm{As}$ are:

$$
\begin{array}{ll}
D_{p} \frac{d \Delta n_{p}}{d x}=S_{p} \Delta n_{p}, & (x=0) \\
\Delta n_{p}=0, & \left(x=x_{j}-x_{p}\right)
\end{array}
$$

The boundary conditions for n-type material, GaAs are:

$D_{n} \frac{d \Delta p_{n}}{d x}=S_{n} \Delta p_{n}, \quad(x=H)$

$\Delta p_{n}=0$,

$$
\left(x=x_{j}+w+x_{n}\right)
$$

where $D_{p, n}$ are diffusion constants of $\mathrm{p}$ - and n-type materials. $p_{n}, n_{p}$ are minority carriers concentration. $\lambda$ and $F$ are the wavelength and photon flux of incident light respectively. $x_{j}$ is the depth of p-layer and $w$ is the width of the MQW layer. $x_{n}$ and $x_{p}$ are the extensions of depletion layer into the n- and p-sides respectively. $H$ is the depth of the structure from front to the back contact. $\tau_{p, n}$ are the lifetimes of the minority carriers. $S_{p, n}$ are the surface recombination velocities. The parameters used for calculations are shown in the following Table:

TABLE I

INPUT PARAMETERS FOR MODELING

\begin{tabular}{|c|c|c|c|c|c|}
\hline Material & $\mathrm{S}\left(\mathrm{cm}^{-1}\right)$ & $\mathrm{L}(\mu \mathrm{m})$ & $\tau(\mathrm{ns})$ & $\mathrm{N}\left(\mathrm{cm}^{-3}\right)$ & Ref. \\
\hline $\mathrm{p}-\mathrm{Al}_{0.37} \mathrm{Ga}_{0.63 \mathrm{v}} \mathrm{As}$ & $10^{6}$ & 2.3 & 3.47 & $1 \times 10^{17}$ & 6 \\
\hline $\mathrm{n}-\mathrm{GaAs}$ & $\infty$ & 2.2 & 7.9 & $1 \times 10^{17}$ & 7 \\
\hline
\end{tabular}

By solving the above equations with their respective boundary conditions, we obtain the photocurrent $J_{n}$ and $J_{p}$. To these, we add the photocurrent contributed from within the depletion region by modifying $J_{d p p}{ }^{8}$ as follows,

$$
\begin{aligned}
J_{d e p}= & q F \exp \left(-x_{j} \alpha_{j}\right)\left[1-\exp \left(-\alpha_{p} x_{p}\right.\right. \\
& \left.\left.-M\left(\alpha_{1} L_{1}+\alpha_{2} L_{2}\right)-\alpha_{n} x_{n}\right)\right]
\end{aligned}
$$

where the first exponential factor is the attenuation of the light through the undepleted $\mathrm{p}$ layer; $\alpha_{p}$ and $\alpha_{p}$ are the absorption coefficients at $\lambda$ of the $\mathrm{p}$ and $\mathrm{n}$ region; $\alpha_{1}$ and $\alpha_{2}$ are the absorption coefficients of the two QW systems; $L_{1}$ and $L_{2}$ are the widths of the QW systems and $M$ the number of QWs.

The assumptions made are that both sides of the junction are taken to be uniform in doping and the reflectance is assumed to be zero for ideal case. The total short-circuit current density then becomes, 
$J_{s c}(\lambda)=J_{n}(\lambda)+J_{p}(\lambda)+J_{d e p}(\lambda)$,

Finally, the spectral response is calculated from the following equation,

$\operatorname{SR}(\lambda)=\frac{J_{s c}(\lambda)}{q F(\lambda)}$

The absorption coefficient we used for bulk $\mathrm{Al}_{\mathrm{X}} \mathrm{Ga}_{1-\mathrm{X}} \mathrm{As}$ and GaAs are published data from ref. 9 and that for $\mathrm{QW}$ system, we calculated from the band mixing QW model ${ }^{10}$.

The energy conversion efficiency, $\eta$, of the solar cell is given by: $\eta=J_{m} V_{m} / P_{i n}$, where $J_{m}$ and $V_{m}$ are the optimum current density and voltage, respectively, which are both given at maximum power. $\boldsymbol{P}_{\text {in }}$ is the incident power per unit area. These are calculated on the basis of a simple superposition model which is also used in Ref. 1 . The current density, $J_{m}$, is given by:

$$
J_{m}=\left|\frac{q n_{p h}\left(E_{g}\right)}{1+\left(k T / q V_{m}\right)}\right|,
$$

where $\mathrm{q}$ is the electric charge; $\mathrm{nph}(\mathrm{Eg})$ is the number of photons with energy greater than the effective bandgap for absorption per unit area per second. $k$ is the Boltzmann constant; $T$ is the temperature, where we used $300 \mathrm{~K}$ in the calculation.

$V_{m}$, is solved from the following equation: $\mathrm{V}_{\mathrm{m}}=\mathrm{V}_{\mathrm{oc}}-\mathrm{kT} \ln \left[1+\left(\mathrm{qV}_{\mathrm{m}} / \mathrm{kT}\right)\right] / \mathrm{q}$, where $V_{o c}$, the open circuit voltage is given by $\mathrm{V}_{\mathrm{c}}=\left[\mathrm{E}_{\mathrm{b}}-\mathrm{kT} \ln \left(\mathrm{A} / \mathrm{J}_{\mathrm{sc}}\right)\right] / \mathrm{q}$. In which, $E_{b}$ is the barrier bandgap, which we assume to be the bulk bandgaps, and $A$ is the parametrization in the dark current density ${ }^{11}$.

\section{RESULTS}

With the addition of the DFQW system, the absorption range of energy is increased, provided that the incident energy is higher than $E_{1-1}$ as depicted on Fig. 2 . Since the resulting bandgap energies vary as the diffusion lengths, we can tailor the bandgaps of the MQW from 1.25 to $1.8 \mathrm{eV}$ by adjusting the diffusion lengths with the use of a mask in the proposed structure. The resulting spectral response of the solar cell with and without the DFQW system are shown in Fig.4. Significant enhancement in spectral response is shown with the adding of the DFQW system.

In Fig.5, we can see the improvement of the energy conversion efficiency over the conventional single bandgap cell. The efficiency is increased by about $5 \%$ in average. In the extreme case, where the bandgap around $1.44 \mathrm{eV}$ which is also the theoretical optimal bandgap for solar cell, the efficiency is even almost $10 \%$ higher.

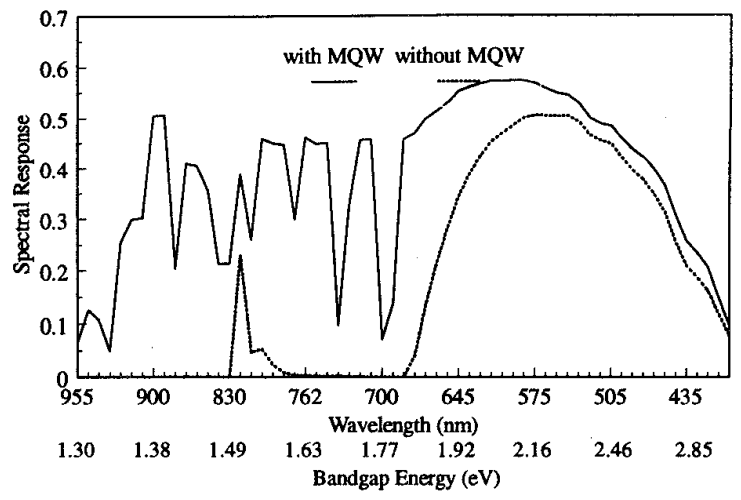

Fig. 4. Spectral response of the DFQW solar cell.

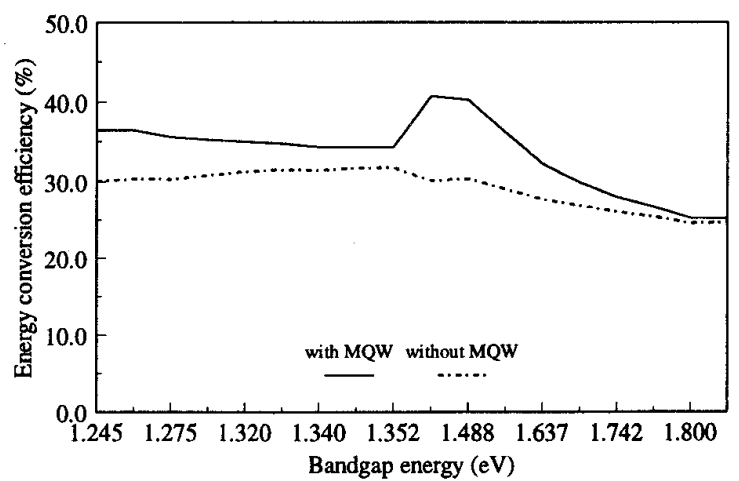

Fig. 5. Comparison of the energy conversion efficiency of the DFQW solar cell and the conventional single bandgap cell.

\section{CONCLUSION}

To conclude, with the spectral response and energy efficiency enhancement demonstrated by our proposed solar cell structure, and in view of the advances in the MBE and MOCVD growth techniques, DFQW solar cell could provide a new approach to the high efficiency solar cell.

\section{ACKNOWLEDGMENT}

One of the authors (Y.C.) would like to acknowledge C.Y. Chan and C.H. Choy for their helpful discussions and suggestions. This work is supported by the HKU-CRCG grant.

\section{REFERENCES}

[1] K.W.J. Barnham and G. Duggan, "A new approach to highefficiency multi-band-gap solar cells", J. Appl. Phys. 67, 3490 (1990). 
[2] K.W.J. Barnham, B. Braun, J. Nelson, M. Paxman, C. Button, J.S. Roberts, and C.T. Foxon, "Short-circuit current and energy efficiency enhancement in a low-dimensional structure photovoltaic device", Appl. Phys. Lett. 59, 135 (1991)

[3] J.Y. Yao, T.G. Andersson and G.L. Dunlop, "Microstructures and critical thicknesses of $\mathrm{In}_{\mathrm{x}} \mathrm{Ga}_{1-\mathrm{x}} \mathrm{As} /$ GaAs strained-layer structures", Semicond. Sci. Technol. 9, 1086 (1994)

[4] E.H. Li, B.L. Weiss and K.S. Chan, "Eigenstate and absorption spectra of interdiffused AlGaAs/GaAs multiple QW structures", J. Appl. Phys. (submitted to Phy. Rev. B)

[5] H.J. Hovel, Semiconductors and Semimetals (Academic, New York, 1975), Vol. 11.

[6] Adachi, Properties of Aluminum Gallium Arsenide (INSPEC, London, 1993).

[7] H.C. Casey, Jr., B.I. Miller and E. J. Pinkas, Appl. Phys., 44, 1281 (1972).

[8] M. Paxman, J. Nelson, B. Braun, J. Connolly, and K.W.J. Barnham, C.T. Foxon and J.S. Roberts, "Modeling the spectral response of the quantum well solar cell", J. Appl. Phys., 74, 614 (1993).

[9] D.E. Aspnes, S.M. Kelso, R.A. Logan, and R. Bhat, "Optical properties of $\mathrm{Al}_{\mathrm{x}} \mathrm{Ga}_{1-\mathrm{x}} \mathrm{As}$ ", J. Appl. Phys., 60, 754 (1986).

[10] E.H. Li, B.L. Weiss, and K.S. Chan, "Effect of interdiffusion on the subbands in an $\mathrm{Al}_{\mathrm{x}} \mathrm{Ga}_{1-\mathrm{x}} \mathrm{As} / \mathrm{GaAs}$ single-quantum-well structure", Phys. Rev. $B, 46,15181$ (1992).

[11] C. H. Henry, "Limiting efficiencies of ideal single and multiple energy gap terrestrial solar cells", J. Appl. Phys., 51, 4494 (1980). 